\title{
Time-independent perturbation theory with Lagrange multipliers
}

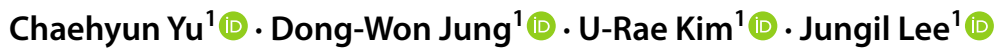

Received: 24 September 2021 / Accepted: 25 October 2021 / Published online: 15 December 2021

(c) The Author(s) 2021

\begin{abstract}
We derive the formulas for the energy and wavefunction of the time-independent Schrödinger equation with perturbation in a compact form. Unlike the conventional approaches based on Rayleigh-Schrödinger or Brillouin-Wigner perturbation theories, we employ a recently developed approach of matrix-valued Lagrange multipliers that regularizes an eigenproblem. The Lagrange-multiplier regularization makes the characteristic matrix for an eigenproblem invertible. After applying the constraint equation to recover the original equation, we find the solutions of the energy and wavefunction consistent with the conventional approaches. This formalism does not rely on an iterative way and the order-by-order corrections are easily obtained by taking the Taylor expansion. The Lagrange-multiplier regularization formalism for perturbation theory presented in this paper is completely new and can be extended to the degenerate perturbation theory in a straightforward manner. We expect that this new formalism is also pedagogically useful to give insights on the perturbation theory in quantum mechanics.
\end{abstract}

Keywords Time-independent perturbation theory $\cdot$ Lagrange multipliers $\cdot$ Eigenvalue problem $\cdot$ Regularization

\section{Introduction}

Stationary states of a quantum-mechanical system are described by the time-independent Schrödinger equation and there are many systems whose exact analytic solutions are known as can be seen in textbooks such as Refs. [1, 2]. However, the exact solution for the time-independent Schrödinger equation for an arbitrary quantum-mechanical system is usually not known or difficult to solve in practice. If the Hamiltonian of a given system deviates by a small amount from that of a system whose exact solutions are known well, then one may find an approximate solution of the given system in terms of the solutions of the well-known system. The small deviation from a well-known system is called perturbation.

The standard time-independent perturbation theory [3] that usually appears in most textbooks like Refs. [1, 2] of quantum mechanics is the Rayleigh-Schrödinger perturbation theory that was first introduced by Schrödinger. This approach is based on the direct calculation of the eigenvalue

Director of Korea Pragmatist Organization for Physics Education $(K P O P \mathscr{E})$ Collaboration.

Jungil Lee

jungil@korea.ac.kr

1 Department of Physics, Korea University, Seoul 02841, Korea with the corresponding eigenvector of a system by explicit series, and, therefore, the formula is very simple at lower orders in the small perturbative parameter. Complications appear in computing corrections at higher orders in the Rayleigh-Schrödinger perturbation theory.

As another perturbation theory, the Brillouin-Wigner perturbation theory has merits against the Rayleigh-Schrödinger counterpart at higher orders because the formulas are much simpler while the first-order corrections of the two theories are the same. However, the weak point of the Brillouin-Wigner theory is that the expansion formula is a function of the energy eigenvalue for the perturbed Hamiltonian, which is unknown, instead of the unperturbed Hamiltonian. A comparison between Rayleigh-Schrödinger and Brillouin-Wigner perturbation theories can be found, for example, in Ref. [4].

Except for the well-known two perturbation theories, several methods to find approximate solutions of a system with small perturbation have been suggested so far. For example, Niblack and Nigam introduced an operator, which turns out to be the operator projecting out the components orthogonal to the unperturbed eigenstate [5]. With the aid of the operator, they obtained the solution for the energy and wavefunction of the system in terms of perturbative potential and unperturbed wavefunction. Lain and Torre found a simple algorithm to derive the formulas 
of both Rayleigh-Schrödinger and Brillouin-Wigner perturbation theories by considering the total corrections to the energy and wavefunction, which are expanded on the eigenfunctions of the unperturbed Hamiltonian [6].

In this paper, we present a new alternative method to find the solution of the perturbative system by employing the Lagrange-multiplier regularization formalism for the eigenproblem, which was suggested in Ref. [7] and successfully applied to the several eigenvalue problems in classical and quantum mechanics. For example, the applications of the Lagrange-multiplier regularization formalism have been made for the normal mode of a loaded string [8] and the inertia tensor of a three-body system [9]. Conventionally, the eigenproblem is solved by finding the eigenvalue, which is the solution of the secular equation, and its corresponding eigenvector by Gaussian elimination. The requirement that the secular equation vanishes implies that the original eigenvalue equation is indeterminate. However, by adding the matrix-valued Lagrange undetermined multipliers to the original eigenvalue equation, we can regularize the indeterminate equation and easily find the eigenvector for a given eigenvalue, which depends on a regularization parameter. The dependence on the regularization parameter can be removed by requiring a constraint equation. This procedure is similar to adding a gauge-fixing term to the gauge-field Lagrangian density in gauge-field theories and turned out to be very powerful to solve several eigenvalue problems in physics [8,9].

We provide a new approach to find the energy and wavefunction of the time-independent Schödinger equation with perturbation by regularizing the eigenvalue equation of the Hamiltonian with matrix-valued Lagrange undetermined multipliers. The regularization makes the characteristic matrix invertible by increasing the number of linearly independent equations. By multiplying the inverse of the regularized characteristic matrix to the regularized eigenvalue equation and imposing the constraint equation that restores the original equation, we find the compact formulas for the energy and wavefunction of the perturbed system. The results agree with those obtained from the conventional method. We expect that this new formalism is pedagogically useful to give insights on the perturbation theory in quantum mechanics.

This paper is organized as follows: In Sect. 2, we define notations involving the Rayleigh-Schrödinger time-independent perturbation theory and review the conventional approach to deal with it. We provide a new Lagrange-multiplier-regularization formalism of determining the energy and wavefunction of the time-independent Schrödinger equation with perturbation in Sect. 3 and conclude in Sect. 4. A rigorous proof of the property of the adjugate matrix which is a crucial part of the formulation is presented in appendix.

\section{Conventional time-independent perturbation theory}

In this section, we first list definitions of the notations that are used in the remainder of this paper involving the Rayleigh-Schrödinger time-independent perturbation theory. Then we review the conventional approach to deal with the Rayleigh-Schrödinger time-independent perturbation theory [3] mainly following the convention given in Ref. [2].

\subsection{Definitions}

The energy eigenket $\left|n^{(0)}\right\rangle$ with the energy eigenvalue $E_{n}^{(0)}$ for the Hamiltonian $H_{0}$ satisfies the eigenvalue equation:

$H_{0}\left|n^{(0)}\right\rangle=E_{n}^{(0)}\left|n^{(0)}\right\rangle$,

which is called the time-independent Schrödinger equation. The integer $n=1,2, \cdots$ stands for the quantum number of the energy eigenket $\left|n^{(0)}\right\rangle$. We assume that the energy eigenstates are nondegenerate. Because $H_{0}$ is Hermitian, $H_{0}^{\dagger}=H_{0}$, the energy eigenvalue $E^{(0)}$ is real, and the eigenkets are orthogonal. The eigenket $\left|n^{(0)}\right\rangle$, which is also called the state ket, is chosen to have the unit normalization:

$\left\langle m^{(0)} \mid n^{(0)}\right\rangle=\delta_{m n}$,

where $\left\langle m^{(0)}\right|$, which is called a bra, is the Hermitian conjugate of the corresponding ket $\left|m^{(0)}\right\rangle$. If we choose $\left\{\left|n^{(0)}\right\rangle \mid n=1,2, \ldots\right\}$ as the basis set of the Hamiltonian $H_{0}$, then the matrix representation of the Hamiltonian can be computed as

$\left(H_{0}\right)_{m n}=\left\langle m^{(0)}\left|H_{0}\right| n^{(0)}\right\rangle$.

According to the Schrödinger equation (1) and the orthogonality relation (2), the Hamiltonian $H_{0}$ of the unperturbed system is a diagonal matrix:

$H_{0}=\operatorname{diag}\left[E_{1}^{(0)} E_{2}^{(0)} \ldots\right]=\left(\begin{array}{lll}E_{1}^{(0)} & & \\ & E_{2}^{(0)} & \\ & & \ddots\end{array}\right)$.

Consider the time-independent Hamiltonian $H$ which is the sum of the unperturbed Hamiltonian $H_{0}$ and a perturbative additional contribution $V$ :

$H=H_{0}+\lambda V$,

where $\lambda$ is a small real parameter and $V$ is Hermitian so that $V^{\dagger}=V$ and $H^{\dagger}=H$. Then the energy eigenket $|n\rangle$ satisfies the eigenvalue equation:

$H|n\rangle=\left(H_{0}+\lambda V\right)|n\rangle=E_{n}|n\rangle$, 
where $E_{n}$ is the energy eigenvalue for the full Hamiltonian $H$ that includes the perturbation. We find the solutions of $E_{n}$ and $|n\rangle$ satisfying Eq. (6). If $\lambda=0$, then $E_{n}=E_{n}^{(0)}$ and $|n\rangle=\left|n^{(0)}\right\rangle$. The perturbation theory finds the Taylor-series expansions of $E_{n}$ and $|n\rangle$ in powers of $\lambda$ about $\lambda=0$.

From the Schrödinger equation (1), it is manifest that $\left(H_{0}-E_{n}^{(0)}\right)\left|n^{(0)}\right\rangle=\mathbb{O}$, where $\mathbb{D}$ is the null ket. Subtracting $E_{n}^{(0)}|n\rangle$ on both sides of the eigenvalue equation (6), we find that

$\left(H_{0}-E_{n}^{(0)}+\lambda V-\Delta_{n} \mathbb{1}\right)|n\rangle=\mathbb{O}$,

where $\mathbb{1}$ is the identity matrix and the energy shift $\Delta_{n}$ is defined by

$\Delta_{n}=E_{n}-E_{n}^{(0)}$.

Note that $\Delta_{n}$ is of order $\lambda^{1}$ or higher because it vanishes as $\lambda \rightarrow 0$.

\subsection{Conventional strategy}

We review the conventional strategy to compute perturbative corrections to the state kets and energy shifts order by order. The coefficients of $\lambda^{k}$ for the energy eigenvalue $E_{n}$ and state ket $|n\rangle$ in the full Schrödinger equation (6) are to be expressed in terms of the corresponding unperturbed values for $E_{k}^{(0)}$ 's and $\left|k^{(0)}\right\rangle$ 's.

Orthogonality of $\left(\lambda V-\Delta_{n} \mathbb{1}\right)|n\rangle$ : We first investigate the orthogonality of $\left(\lambda V-\Delta_{n} \mathbb{1}\right)|n\rangle$ with respect to the unperturbed ket $\left|n^{(0)}\right\rangle$. By applying the unperturbed bra $\left\langle n^{(0)}\right|$ to the left of the full Schrödinger equation (7) and taking the Hermitian conjugate of the unperturbed eigenvalue equation (1), we find that $\left(\lambda V-\Delta_{n}\right)|n\rangle$ is orthogonal to $\left|n^{(0)}\right\rangle$ :

$\left\langle n^{(0)}\left|\left(\lambda V-\Delta_{n} \mathbb{1}\right)\right| n\right\rangle=0$.

This immediately yields the identity for the energy shift $\Delta_{n}$ :

$\Delta_{n}=\frac{\lambda\left\langle n^{(0)}|V| n\right\rangle}{\left\langle n^{(0)} \mid n\right\rangle}=\lambda\left\langle n^{(0)}|V| n\right\rangle$,

where we have set the normalization for $|n\rangle$ such that

$\left\langle n^{(0)} \mid n\right\rangle=\left\langle n^{(0)} \mid n^{(0)}\right\rangle=1, \quad\left\langle n^{(0)} \mid m^{(0)}\right\rangle=\delta_{n m}$.

\subsubsection{Requirement of renormalization}

We emphasize that the normalization condition $\left\langle n^{(0)} \mid n\right\rangle=1$ is not required but is chosen for convenience. However, this choice is crucial to simplify the intermediate steps significantly because the condition disallows any overlap between the unperturbed state $\left|n^{(0)}\right\rangle$ and the perturbative corrections $\lambda^{k}\left|n^{(k)}\right\rangle$ to all orders of $\lambda^{k}$ for $k \geq 1$. While this prescription provides us with a convenience in the intermediate steps, it brings in an additional procedure as a payback at the end of the calculation which is called the renormalization. The reason is that the choice of the normalization $\left\langle n^{(0)} \mid n\right\rangle=\left\langle n^{(0)} \mid n^{(0)}\right\rangle=1$ results in $\langle n \mid n\rangle \neq 1$ because $|n\rangle$ does acquire nonvanishing components orthogonal to $\left|n^{(0)}\right\rangle$ due to perturbation. Therefore, it is required to renormalize $|n\rangle$ not now but at the end of the calculation by replacing $|n\rangle$ as

$|n\rangle \rightarrow \frac{|n\rangle}{\sqrt{\langle n \mid n\rangle}}$.

The renormalization procedure must be postponed until we complete the calculation for the perturbative corrections for $\left|n^{(k)}\right\rangle$ to the last order that we concern.

\subsubsection{Perturbative expansion of $|n\rangle$}

The eigenket can be expanded in powers of $\lambda$ as

$|n\rangle=\left|n^{(0)}\right\rangle+\sum_{k=1}^{\infty} \lambda^{k}\left|n^{(k)}\right\rangle$.

The coefficient kets $\left|n^{(k)}\right\rangle$ are independent of $\lambda$. This expansion is consistent with the normalization in Eq. (11) and

$\left\langle n^{(0)} \mid n^{(k)}\right\rangle=0, \quad k \neq 0$.

Thus we confirm that the choice of the normalization for $\left\langle n^{(0)} \mid n\right\rangle=1$ in Eq. (11) greatly simplifies the intermediate computation because of the orthogonality relation in Eq. (14).

\subsubsection{Perturbative expansion of $\Delta_{n}^{(k)}$}

Note that $\Delta_{n}$ can be expanded in a power series of $\lambda$ as

$\Delta_{n}=\lambda \Delta_{n}^{(1)}+\lambda^{2} \Delta_{n}^{(2)}+\cdots=\sum_{k=1}^{\infty} \lambda^{k} \Delta_{n}^{(k)}$,

which starts from the order of $\lambda^{1}$ due to the consistency in the limit of $\lambda \rightarrow 0$. Substituting Eqs. (13) and (15) into the second formula of Eq. (10) with the condition $\left\langle n^{(0)} \mid n\right\rangle=1$, we find that the coefficient of $\lambda^{k}$ in the energy shift $\Delta_{n}$ is

$\Delta_{n}^{(k)}=\left\langle n^{(0)}|V| n^{(k-1)}\right\rangle, \quad k=1,2, \ldots$,

which implies that the $k$ th correction to the energy shift is determined by the $(k-1)$ th-order correction $\left|n^{(k-1)}\right\rangle$ to the eigenket.

Operator $\phi_{n} /\left(E_{n}^{(0)}-H_{0}\right)$ : According to Eq. (9), $\left(\lambda V-\Delta_{n} \mathbb{1}\right)|n\rangle$ is always orthogonal to $\left|n^{(0)}\right\rangle$. Therefore, $\left(E_{n}^{(0)}-H_{0}\right)^{-1}\left(\lambda V-\Delta_{n} \mathbb{1}\right)$ is a well-defined matrix because the denominator never vanishes. Thus we define 


$$
\begin{aligned}
\frac{\phi_{n}}{E_{n}^{(0)}-H_{0}}\left(\lambda V-\Delta_{n} \mathbb{1}\right) & \equiv\left(E_{n}^{(0)}-H_{0}\right)^{-1} \phi_{n}\left(\lambda V-\Delta_{n} \mathbb{1}\right) \\
& =\left(E_{n}^{(0)}-H_{0}\right)^{-1}\left(\lambda V-\Delta_{n} \mathbb{1}\right),
\end{aligned}
$$

where $\phi_{n}$ is the projection operator that projects out the components orthogonal to $\left|n^{(0)}\right\rangle$ :

$\phi_{n}=\mathbb{1}-\left|n^{(0)}\right\rangle\left\langle n^{(0)}\left|=\sum_{k \neq n}\right| k^{(0)}\right\rangle\left\langle k^{(0)}\right|$.

Trivial properties of this projection operator are

$\phi_{n}\left|n^{(0)}\right\rangle=\mathbb{D}, \quad \phi_{n}^{k}=\phi_{n}, \quad k=1,2, \ldots$,

which denote the orthogonality and idempotent property, respectively.

\subsection{Result for the eigenket}

The eigenket $|n\rangle$ can be expanded by respecting the normalization (11) and the eigenvalue equation (7), and by applying the identity (17) as

$|n\rangle=\left|n^{(0)}\right\rangle+\frac{\phi_{n}}{E_{n}^{(0)}-H_{0}}\left(\lambda V-\Delta_{n} \mathbb{1}\right)|n\rangle$,

where each term on the right side represents the component proportional and orthogonal to the unperturbed eigenket $\left|n^{(0)}\right\rangle$, respectively. Moving the last term on the right side to the left and solving $|n\rangle$, we find that

$|n\rangle=\left[\mathbb{1}-\frac{\phi_{n}}{E_{n}^{(0)}-H_{0}}\left(\lambda V-\Delta_{n} \mathbb{1}\right)\right]^{-1}\left|n^{(0)}\right\rangle$.

A note should be added in regarding the expression in Eq. (21). The conventional approaches that can be seen for example in Refs. [1,2] rely on cumbersome iterative procedure to find the order-by-order expression for $\left|n^{(k)}\right\rangle$. This procedure is simplified into a one-step operation in Eq. (21). The contribution of the second term in the brackets is of order $\lambda^{1}$ or higher according to Eqs. (10) and (13). If we assume that the contribution is small by taking $\lambda \ll 1$, then we can make a Taylor-series expansion of the inverse operator as

$|n\rangle=\left\{\sum_{k=0}^{\infty}\left[\frac{\phi_{n}}{E_{n}^{(0)}-H_{0}}\left(\lambda V-\Delta_{n} \mathbb{1}\right)\right]^{k}\right\}\left|n^{(0)}\right\rangle$.

The expansion of the operator from Eqs. (21)-(22) is similar to the expansion of the geometric series: $1 /(1-r)=1+r+r^{2}+\cdots=\sum_{k=0}^{\infty} r^{k}$ for $|r|<1$.
One should take special care of dealing with the order-byorder computation of the perturbative contribution. While $\lambda V$ has the contribution of order $\lambda$ only, $\Delta_{n}$ does have contributions of order $\lambda^{k}$ for all possible values for $k=1,2, \cdots$. Substituting Eq. (16) into Eq. (15) and substituting this $\Delta_{n}$ into Eq. (22), we find the resultant power-series expression for $|n\rangle$ to all orders in $\lambda$ :

$|n\rangle=\left\{\sum_{k=0}^{\infty}\left[\frac{\phi_{n}}{E_{n}^{(0)}-H_{0}}\left(\lambda V-\mathbb{1} \sum_{\ell=1}^{\infty} \lambda^{\ell}\left\langle n^{(0)}|V| n^{(\ell-1)}\right\rangle\right)\right]^{k}\right\}\left|n^{(0)}\right\rangle$.

Substituting Eq. (13) on the left side and comparing each term of order $\lambda^{k}$ on both sides, we find the eigenket $|n\rangle$ in a power series of $\lambda$. In practice the series expansion is truncated at a certain order in $\lambda$. The result given in Eq. (23) is the consequence of the normalization choice in Eq. (11). Therefore, we must renormalize the state ket $|n\rangle$ following Eq. (12) as we have stated in the previous subsection.

Here, we have assumed that the interaction potential $\lambda V$ is small. In general, $\lambda V$ is not necessarily small. It is worthwhile pointing out that the formula (21) still holds even for a fairly large potential. Then, one can find the eigenket $|n\rangle$ from Eq. (21) if the convergence of the expansion is reasonably good.

\subsection{Order-by-order formulas}

We list the resultant formulas for the perturbative corrections to the kets and energy shifts to order $\lambda^{3}$. The zerothorder contribution is the identity $\left|n^{(0)}\right\rangle=\left|n^{(0)}\right\rangle$ because the first term in the sum on the right side of Eq. (23) is just $\mathbb{1}\left|n^{(0)}\right\rangle=\left|n^{(0)}\right\rangle$.

\subsubsection{State kets}

The first-order contribution can be read off as

$\left|n^{(1)}\right\rangle=\frac{\phi_{n}}{E_{n}^{(0)}-H_{0}}\left(V-\mathbb{1}\left\langle n^{(0)}|V| n^{(0)}\right\rangle\right)\left|n^{(0)}\right\rangle=\frac{\phi_{n}}{E_{n}^{(0)}-H_{0}} V\left|n^{(0)}\right\rangle$,

where we have made use of the identity $\phi_{n}\left|n^{(0)}\right\rangle=0$. The second-order contribution can be extracted as

$$
\begin{aligned}
\left|n^{(2)}\right\rangle= & \frac{\phi_{n}}{E_{n}^{(0)}-H_{0}} V \frac{\phi_{n}}{E_{n}^{(0)}-H_{0}} V \\
& \left.\quad-\frac{\phi_{n}}{E_{n}^{(0)}-H_{0}}\left\langle n^{(0)}|V| n^{(0)}\right\rangle \mathbb{1} \frac{\phi_{n}}{E_{n}^{(0)}-H_{0}} V\right)\left|n^{(0)}\right\rangle .
\end{aligned}
$$

Note that the contributions of $k=0$ and 1 of the summation in Eq. (23) do not contribute. In these contributions the 
second-order $\left(\lambda^{2}\right)$ contribution is at $\ell=2$ in the summation over $\ell$ for $k=1$. However, the contribution is proportional to $\phi_{n}$ and, therefore, vanishes after acting on the ket $\left|n^{(0)}\right\rangle$. The $k=2$ contribution is expressed as the square of the operator in the brackets in Eq. (23), where $\ell$ and $\ell^{\prime}$ are used for the two summation indices in the parentheses in Eq. (23) in operating order. Among the $k=2$ contributions, the $\ell^{\prime}=1$ contribution vanishes because it is proportional to $\left[\phi_{n} /\left(E_{n}^{(0)}-H_{0}\right)\right] \mathbb{1}$ with some prefactors. The first term in the parentheses in Eq. (25) is for $k=2$ and $\ell=\ell^{\prime}=0$, while the second term is for $k=2, \ell=1$, and $\ell^{\prime}=0$.

In a similar manner, we can read off the third-order contribution in a straightforward way:

$$
\begin{aligned}
\left|n^{(3)}\right\rangle= & \frac{\phi_{n}}{E_{n}^{(0)}-H_{0}} V \frac{\phi_{n}}{E_{n}^{(0)}-H_{0}} V \frac{\phi_{n}}{E_{n}^{(0)}-H_{0}} V \\
& -\frac{\phi_{n}}{E_{n}^{(0)}-H_{0}}\left\langle n^{(0)}|V| n^{(0)}\right\rangle \mathbb{1} \frac{\phi_{n}}{E_{n}^{(0)}-H_{0}} V \frac{\phi_{n}}{E_{n}^{(0)}-H_{0}} V \\
& -\frac{\phi_{n}}{E_{n}^{(0)}-H_{0}} V \frac{\phi_{n}}{E_{n}^{(0)}-H_{0}}\left\langle n^{(0)}|V| n^{(0)}\right\rangle \mathbb{1} \frac{\phi_{n}}{E_{n}^{(0)}-H_{0}} V \\
& -\frac{\phi_{n}}{E_{n}^{(0)}-H_{0}}\left\langle n^{(0)}|V| n^{(0)}\right\rangle \mathbb{1} \frac{\phi_{n}}{E_{n}^{(0)}-H_{0}}\left\langle n^{(0)}|V| n^{(0)}\right\rangle \mathbb{1} \frac{\phi_{n}}{E_{n}^{(0)}-H_{0}} V \\
& \left.-\frac{\phi_{n}}{E_{n}^{(0)}-H_{0}}\left\langle n^{(0)}\left|V \frac{\phi_{n}}{E_{n}^{(0)}-H_{0}} V\right| n^{(0)}\right\rangle \mathbb{1} \frac{\phi_{n}}{E_{n}^{(0)}-H_{0}} V\right)\left|n^{(0)}\right\rangle,
\end{aligned}
$$

where we have made use of Eq. (24). The formulas for the state kets follow the normalization in Eq. (11) that are not properly normalized. Thus they require the renormalization as is shown in Eq. (12) once the truncation of the perturbative series is determined at a certain finite order.

\subsubsection{Energy shifts}

The energy shift can be computed to order $\lambda^{3}$ by substituting $\left|n^{(k)}\right\rangle$ in Eqs. (24) and (25) into Eq. (16) as

$\Delta_{n}^{(1)}=\left\langle n^{(0)}|V| n^{(0)}\right\rangle$,

$$
\begin{aligned}
\Delta_{n}^{(2)} & =\left\langle n^{(0)}|V| n^{(1)}\right\rangle=\left\langle n^{(0)}\left|V \frac{\phi_{n}}{E_{n}^{(0)}-H_{0}} V\right| n^{(0)}\right\rangle \\
& =\sum_{k \neq n} \frac{\left|V_{n k}\right|^{2}}{E_{n}^{(0)}-E_{k}^{(0)}},
\end{aligned}
$$

$$
\begin{aligned}
\Delta_{n}^{(3)}= & \left\langle n^{(0)}|V| n^{(2)}\right\rangle=\left\langle n^{(0)}\left|V \frac{\phi_{n}}{E_{n}^{(0)}-H_{0}} V \frac{\phi_{n}}{E_{n}^{(0)}-H_{0}} V\right| n^{(0)}\right\rangle \\
& -\left\langle n^{(0)}\left|V \frac{\phi_{n}}{E_{n}^{(0)}-H_{0}}\left\langle n^{(0)}|V| n^{(0)}\right\rangle \frac{\phi_{n}}{E_{n}^{(0)}-H_{0}} V\right| n^{(0)}\right\rangle \\
= & \sum_{k \neq n} \sum_{\ell \neq n} \frac{V_{n k} V_{k \ell} V_{\ell n}}{\left(E_{n}^{(0)}-E_{k}^{(0)}\right)\left(E_{n}^{(0)}-E_{\ell}^{(0)}\right)}-\sum_{k \neq n} \frac{V_{n n}\left|V_{n k}\right|^{2}}{\left(E_{n}^{(0)}-E_{k}^{(0)}\right)^{2}},
\end{aligned}
$$

where we have made use of the fact that $V$ is a Hermitian operator $V^{\dagger}=V$. The matrix elements for $V$ are defined in terms of the unperturbed state kets as

$V_{n m}=\left\langle n^{(0)}|V| m^{(0)}\right\rangle$.

Thus $V_{i j} V_{j i}=V_{i j} V_{i j}^{\star}=\left|V_{i j}\right|^{2}$ for any $i$ and $j$.

\section{Lagrange-multiplier approach}

In this section, we present an alternative derivation of the perturbative-series expansions displayed in Eqs. (21) and (22) involving the full Hamiltonian $H=H_{0}+\lambda V$. Our derivation is completely independent of the conventional approach described in Sect. 2. Instead, we regularize the eigenvalue equation with matrix-valued Lagrange undetermined multipliers. In Ref. [9], the Lagrange-multiplier-regularization formalism was first introduced to solve a system of indeterminate linear equations. A generalized version of the formalism applicable to the eigenproblem was developed in Ref. [7]. Very recently, the Lagrange-multiplier-regularization formalism is further developed to include a convenient adjugate representation in Ref. [8].

\subsection{Lagrange-multiplier regularization}

The original unperturbed system satisfies the Schrödinger equation (1). In perturbation theory, one assumes that the exact solution for the unperturbed eigenvalue equation is known. However, in this work we begin with solving the original unperturbed eigenvalue equation by applying the Lagrangeundetermined-multiplier regularization. This is a good example of demonstrating how the Lagrange-multiplier regularization works. Firstly, we observe that the eigenvalue equation (1) is indeterminate. In general, a Lagrange undetermined multiplier introduces a new degree of freedom that the given system is lacking due to a constraint. Intrinsically, the orthogonality between the characteristic matrix and the eigenvector corresponds to such a constraint. Thus the characteristic matrix is lacking the information along the direction parallel to the eigenvector. The Lagrange-multiplier regularization resurrects the lacking degree of freedom of the characteristic equation in a similar spirit as a usual Lagrange multiplier in Lagrangian mechanics does. Once a valid regularization is achieved, one can find the inverse transformation of the equation to find the regularized solution. The right side of the eigenvalue equation must be regularized simultaneously because the sole regularization of the left side leads to a trivial null vector. A valid regularization has the boundary condition that the regularized equation reproduces the original equation if we turn off the regularization parameter at any stage. Thus the solution to the 
original indeterminate equation is restored if we impose the constraint equation into the regularized solution.

The Schrödinger equation (1) for the unperturbed system is manifestly indeterminate because the determinant of the characteristic matrix is vanishing: $\mathscr{D e t}\left[H_{0}-E_{n}^{(0)} \mathbb{1}\right]=0$. The reason is that every row of the characteristic matrix $H_{0}-E_{n}^{(0)} \mathbb{1}$ is linearly independent of the eigenket $\left|n^{(0)}\right\rangle$. To make the linear equation solvable, we regularize the characteristic matrix by adding a projection operator $\left|n^{(0)}\right\rangle\left\langle n^{(0)}\right|$ multiplied by the regularization parameter $\alpha$, which is in general a complex number and vanishes if we restore the original equation. The following regularization does not modify Eq. (1) at all for any complex number $\alpha$ :

$\left(H_{0}-E_{n}^{(0)}+\alpha\left|n^{(0)}\right\rangle\left\langle n^{(0)}\right|\right)\left|n^{(0)}\right\rangle=\alpha\left|n^{(0)}\right\rangle, \quad \alpha \in \mathbb{C}$,

where $\mathbb{C}$ is the set of complex numbers. In fact, the eigenket $\left|n^{(0)}\right\rangle$ is unknown yet and must be found as a solution of Eq. (1). Hence, the regularized equation (31) is practically useless. We make a further tuning that replaces $\left|n^{(0)}\right\rangle$ with an arbitrary vector. This vector must have the component along $\left|n^{(0)}\right\rangle$. This is equivalent to the replacement of $\left|n^{(0)}\right\rangle\left\langle n^{(0)}\right|$ with $\mathbb{1}$, which is the simplest choice. Due to the modification of the left side, $\left|n^{(0)}\right\rangle$ on the right side must be modified as an arbitrary ket $|c\rangle$. However, it turns out that only the component parallel to $\left|n^{(0)}\right\rangle$ survives after applying the constraint equation as we will show later. This is a valid fine tuning because the identity matrix does project both longitudinal and transverse directions with respect to whatsoever $\left|n^{(0)}\right\rangle$ is: $\mathbb{1}\left|n^{(0)}\right\rangle\left\langle n^{(0)}|=| n^{(0)}\right\rangle\left\langle n^{(0)}\right|$ and $\mathbb{1}\left(\mathbb{1}-\left|n^{(0)}\right\rangle\left\langle n^{(0)}\right|\right)=\mathbb{1}-\left|n^{(0)}\right\rangle\left\langle n^{(0)}\right|$. Then we arrive at the regularized eigenvalue equation:

$\left(H_{0}-E_{n}^{(0)} \mathbb{1}+\alpha \mathbb{1}\right)\left|n^{(0)}(\alpha)\right\rangle=\alpha|c\rangle$,

where the matrix-valued Lagrange undetermined multipliers are $\mathbb{1}$ in $\alpha \mathbb{1}$ on the left side and $|c\rangle$ on the right side. Note that the regularized ket $\left|n^{(0)}(\alpha)\right\rangle$ does acquire the dependence on the regularization parameter $\alpha$ and the original equation (1) is restored as $\alpha \rightarrow 0$. Having resurrected the lacking degree of freedom in the characteristic matrix $H_{0}-E_{n}^{(0)} \mathbb{1}+\alpha \mathbb{1}$, we are able to solve $\left|n^{(0)}(\alpha)\right\rangle$ by making an ordinary inverse linear transformation of Eq. (32). In principle, one can use any other matrix-valued multiplier $\mathbb{B}$ instead of $\mathbb{1}$ as long as $H_{0}-E_{n}^{(0)} \mathbb{1}+\alpha \mathbb{B}$ is invertible. Thus the explicit form of $\left|n^{(0)}(\alpha)\right\rangle$ depends on the multiplier. One might worry about a possible scheme dependence of the regularization coming from the choice in the undetermined multiplier of either $\mathbb{1}$ or $\mathbb{B}$. However, the boundary condition that reproduces the original equation prohibits the regularization scheme dependence as $\alpha \rightarrow 0$.
The eigenvalue $E_{n}^{(0)}$ is the solution for the secular equation

$\mathscr{D}=\mathscr{D e t}\left[H_{0}-E_{n}^{(0)} \mathbb{1}\right]=0$,

where $\mathscr{D}$ is called the secular determinant. The corresponding regularized secular determinant is not vanishing any more if $\alpha \neq 0$ :

$\mathscr{D}(\alpha)=\mathscr{D e t}\left[H_{0}-E_{n}^{(0)} \mathbb{1}+\alpha \mathbb{1}\right] \propto \alpha \neq 0$.

If every eigenvalue is distinguished from any other eigenvalues, then we say the system is non-degenerate. If the system is non-degenerate, then $\mathscr{D}(\alpha)$ has the asymptotic behavior proportional to $\alpha$ as $\alpha \rightarrow 0$. Thus, the parameter $\alpha$ makes the matrix $\left(H_{0}-E_{n}^{(0)} \mathbb{1}+\alpha \mathbb{1}\right)$ invertible: $\left(H_{0}-E_{n}^{(0)} \mathbb{1}+\alpha \mathbb{1}\right)^{-1}$ exists. Once $E_{n}^{(0)}$ is known from the secular equation, one can solve $\left|n^{(0)}\right\rangle$ by multiplying the inverse $\left(H_{0}-E_{n}^{(0)} \mathbb{1}+\alpha \mathbb{1}\right)^{-1}$ to the left of the regularized eigenvalue equation (32). The result is

$\left|n^{(0)}(\alpha)\right\rangle=\alpha\left(H_{0}-E_{n}^{(0)} \mathbb{1}+\alpha \mathbb{1}\right)^{-1}|c\rangle$.

It is remarkable that $\alpha\left(H_{0}-E_{n}^{(0)} \mathbb{1}+\alpha \mathbb{1}\right)^{-1}$ is analytic at $\alpha=0$ although $\left(H_{0}-E_{n}^{(0)} \mathbb{1}+\alpha \mathbb{1}\right)^{-1}$ has a singularity proportional to $1 / \alpha$ as $\alpha \rightarrow 0$. The reason is that $\mathscr{D}(\alpha) \propto \alpha$ as $\alpha \rightarrow 0$. Since $|c\rangle$ is an arbitrary ket, one must rescale $|c\rangle$ by a constant factor after finishing the calculation to require a consistent normalization. To gain a more concrete insight on the $\alpha$-dependence of the eigenket after the regularization, we separate $\left|n^{(0)}(\alpha)\right\rangle$ into two components with and without the $\alpha$ dependence. Note that we have the initial condition:

$\left|n^{(0)}(0)\right\rangle=\left|n^{(0)}\right\rangle$.

The analyticity on the right side of Eq. (35) guarantees that $\left|n^{(0)}(\alpha)\right\rangle$ can be expanded about $\alpha=0$ as

$\left|n^{(0)}(\alpha)\right\rangle=\left|n^{(0)}\right\rangle+\alpha \Delta\left|n^{(0)}(\alpha)\right\rangle$,

where the second term $\alpha \Delta\left|n^{(0)}(\alpha)\right\rangle$ vanishes as $\alpha \rightarrow 0$. Here, the operator symbol $\Delta$ in Eq. (37) should be distinguished from the energy shift $\Delta_{n}$ defined in Eq. (8). Substituting Eq. (37) into (35), we find that

$\left|n^{(0)}\right\rangle=\alpha\left(H_{0}-E_{n}^{(0)} \mathbb{1}+\alpha \mathbb{1}\right)^{-1}|c\rangle-\alpha \Delta\left|n^{(0)}(\alpha)\right\rangle$.

Since the left side is free of $\alpha$, it is manifest that the $\alpha$ dependence on the right side cancels completely. Furthermore, Eq. (38) holds for any value of $\alpha$. The simplest choice is to take $\alpha=0$ and the second term on the right side vanishes in the limit of $\alpha \rightarrow 0$.

According to Ref. [8], the matrix on the right side of Eq. (38) is actually the adjugate matrix for the 
original characteristic matrix in the limit of $\alpha \rightarrow 0$ if $H_{0}$ is not degenerate:

$$
\begin{aligned}
\left|n^{(0)}\right\rangle & =\lim _{\alpha \rightarrow 0} \frac{\alpha}{\mathscr{D}(\alpha)} \times \mathscr{D}(\alpha)\left(H_{0}-E_{n}^{(0)} \mathbb{1}+\alpha \mathbb{1}\right)^{-1}|c\rangle \\
& =\operatorname{adj}\left(H_{0}-E_{n}^{(0)} \mathbb{1}\right)|c\rangle,
\end{aligned}
$$

where we have absorbed the finite constant factor $\lim _{\alpha \rightarrow 0} \alpha / \mathscr{D}(\alpha)$ into $|c\rangle$ by rescaling the normalization. Here, $\mathscr{D}(\alpha)$ is defined in Eq. (34). The adjugate $\operatorname{adj}(\mathbb{A})$ of a matrix $\mathbb{A}$ is the transpose of the cofactor matrix $\mathbb{C}$ whose $i j$ element is the $i j$ minor $\mathscr{M}_{i j}$ of the matrix $\mathbb{A}$ multiplied by $(-1)^{i+j}$. The $i j$ minor $\mathscr{M}_{i j}$ of the matrix $\mathbb{A}$ is the determinant of the submatrix of $\mathbb{A}$ in which the $i$ th row and the $j$ th column are eliminated from $\mathbb{A}$. The matrix representation for the adjugate matrix $\operatorname{adj}\left(H_{0}-E_{n}^{(0)} \mathbb{1}\right)$ is quite simple in the basis set $\left\{\left|n^{(0)}\right\rangle \mid n=1,2, \ldots\right\}$ for the original Hamiltonian $H_{0}$. The reason is that $H_{0}$ is diagonal with the eigenkets: all of the matrix elements are vanishing except for the $n n$ element whose value is given by

$\left\langle n^{(0)}\left|\operatorname{adj}\left(H_{0}-E_{n}^{(0)} \mathbb{1}\right)\right| n^{(0)}\right\rangle=\prod_{k \neq n}\left(E_{k}^{(0)}-E_{n}^{(0)}\right)$.

As a result, the limiting value of the operator $\mathscr{D}(\alpha)\left(H_{0}-E_{n}^{(0)} \mathbb{1}+\alpha \mathbb{1}\right)^{-1}$ as $\alpha \rightarrow 0$ is actually the projection operator that selects the component parallel to $\left|n^{(0)}\right\rangle$ up to an overall constant factor of $\prod_{k \neq n}\left(E_{k}^{(0)}-E_{n}^{(0)}\right)$ :

$$
\begin{aligned}
\lim _{\alpha \rightarrow 0} \mathscr{D}(\alpha)\left(H_{0}-E_{n}^{(0)} \mathbb{1}+\alpha \mathbb{1}\right)^{-1} & =\operatorname{adj}\left(H_{0}-E_{n}^{(0)} \mathbb{1}\right) \\
& =\left|n^{(0)}\right\rangle\left\langle n^{(0)}\right| \prod_{k \neq n}\left(E_{k}^{(0)}-E_{n}^{(0)}\right) .
\end{aligned}
$$

This is a manifestation of the primitive regularized Eq. (31). In appendix 1, we present a rigorous proof of the identity (41). By making use of the completeness,

$\mathbb{1}=\sum_{m=1}^{\infty}\left|m^{(0)}\right\rangle\left\langle m^{(0)}\right|$

of the Hilbert space spanned by the eigenkets, we can express $\left|n^{(0)}\right\rangle$ in Eq. (39) into the form

$$
\begin{aligned}
\left|n^{(0)}\right\rangle & =\sum_{m=1}^{\infty} \operatorname{adj}\left(H_{0}-E_{n}^{(0)} \mathbb{1}\right)\left|m^{(0)}\right\rangle\left\langle m^{(0)} \mid c\right\rangle \\
& =\left|n^{(0)}\right\rangle\left\langle n^{(0)} \mid c\right\rangle \prod_{k \neq n}\left(E_{k}^{(0)}-E_{n}^{(0)}\right),
\end{aligned}
$$

where we have made use of the identity (41) and the orthonormal relation for the unperturbed state kets in Eq. (11). The normalization can be corrected consistently by choosing $\left\langle n^{(0)} \mid c\right\rangle=1 / \prod_{k \neq n}\left(E_{k}^{(0)}-E_{n}^{(0)}\right)$. This leads to a trivial identity $\left|n^{(0)}\right\rangle=\left|n^{(0)}\right\rangle$.

\subsection{Finding $|n\rangle$ with Lagrange multipliers}

\subsubsection{Regularized equation}

The eigenvalue equation (7) is an indeterminate equation: the characteristic determinant $\mathscr{D} e t\left[H_{0}-E_{n}^{(0)}+\lambda V-\Delta_{n}\right]$ for the perturbed system is vanishing and, therefore, the inverse $\left(H_{0}-E_{n}^{(0)}+\lambda V-\Delta_{n}\right)^{-1}$ does not exist. Following the approaches of Refs. [7, 8] and making use of the results in the previous subsection, we again carry out the Lagrange-multiplier regularization of the eigenproblem in Eq. (7) for the perturbed system with the regularization parameter $\alpha$ as

$\left(H_{0}-E_{n}^{(0)} \mathbb{1}+\lambda V-\Delta_{n} \mathbb{1}+\alpha \mathbb{1}\right)|n(\alpha)\rangle=\alpha|c\rangle$,

where $\mathbb{1}$ in the term $\alpha \mathbb{1}$ and the arbitrary constant ket $|c\rangle$ are Lagrange multipliers. Note that the $\alpha$ dependence in the ket $|n(\alpha)\rangle$ disappears as we take the limit $\alpha \rightarrow 0$.

\subsubsection{Factorization of the operator}

We can in principle carry out the regularization procedure for the full Hamiltonian in a straightforward way. However, it is more efficient to make use of the findings from the unperturbed case which leads to a systematic reduction. Therefore, we are to employ an additional procedure in which the full operator is expressed as the product of the original operator without perturbation and the remainder. The factorization is a remarkable advantage of the Lagrange-multiplier-regularization formalism that reduces the intermediate steps significantly. Such a factorization is disallowed in the original equation due to the nonexistence of the inverse transformation. It is convenient to make use of the results in Sect. 3.1 for the unperturbed case. Thus we pull out the matrix $\left(H_{0}-E_{n}^{(0)} \mathbb{1}+\alpha \mathbb{1}\right)$ from Eq. (44) as

$$
\begin{aligned}
& \left(H_{0}-E_{n}^{(0)} \mathbb{1}+\alpha \mathbb{1}\right)\left[\mathbb{1}+\left(H_{0}-E_{n}^{(0)} \mathbb{1}+\alpha \mathbb{1}\right)^{-1}\left(\lambda V-\Delta_{n} \mathbb{1}\right)\right]|n(\alpha)\rangle \\
& =\alpha|c\rangle .
\end{aligned}
$$

If it were not for the Lagrange-multiplier-regularization procedure, we should be unable to find the inverse transformation. Following the strategy in the previous subsection, we separate the $\alpha$-dependent part of $|n(\alpha)\rangle$ as

$|n(\alpha)\rangle=|n\rangle+\alpha \Delta|n(\alpha)\rangle$

where $\alpha \Delta|n(\alpha)\rangle$ vanishes as $\alpha \rightarrow 0$. Substituting Eq. (46) into Eq. (45), we find that 


$$
\begin{aligned}
& \left(H_{0}-E_{n}^{(0)} \mathbb{1}+\alpha \mathbb{1}\right)\left[\mathbb{1}+\left(H_{0}-E_{n}^{(0)} \mathbb{1}+\alpha \mathbb{1}\right)^{-1}\left(\lambda V-\Delta_{n} \mathbb{1}\right)\right]|n\rangle \\
& =\alpha|c\rangle-\alpha M(\alpha) \Delta|n(\alpha)\rangle,
\end{aligned}
$$

where $M(\alpha)$ is an analytic matrix at $\alpha=0$ which is defined by

$$
\begin{aligned}
M(\alpha) \equiv & \left(H_{0}-E_{n}^{(0)} \mathbb{1}+\alpha \mathbb{1}\right) \\
& \times\left[\mathbb{1}+\left(H_{0}-E_{n}^{(0)} \mathbb{1}+\alpha \mathbb{1}\right)^{-1}\left(\lambda V-\Delta_{n} \mathbb{1}\right)\right] .
\end{aligned}
$$

The factorized form in Eq. (47) reveals that the singularity is regularized by the $\alpha$-dependent term $\alpha \mathbb{1}$ and the remaining operator is free of singularity as $\alpha \rightarrow 0$ because of the following reason: As we have discussed during the derivation from Eqs. (20) to (21), we can make use of the fact that $\left(\lambda V-\Delta_{n} \mathbb{1}\right)|n\rangle$ is orthogonal to $\left|n^{(0)}\right\rangle$. Thus the result is invariant under the insertion of the projection operator $\phi_{n}$ defined in Eq. (18) in front of $\left(\lambda V-\Delta_{n} \mathbb{1}\right)|n\rangle$ :

$\left(\lambda V-\Delta_{n} \mathbb{1}\right)|n\rangle=\phi_{n}\left(\lambda V-\Delta_{n} \mathbb{1}\right)|n\rangle$.

The insertion, however, makes it clear that the inverse of $\left(H_{0}-E_{n}^{(0)} \mathbb{1}+\alpha \mathbb{1}\right)^{-1}$ still exists even in the limit $\alpha \rightarrow 0$. Then we find that

$$
\begin{aligned}
& \left(H_{0}-E_{n}^{(0)} \mathbb{1}+\alpha \mathbb{1}\right)\left[\mathbb{1}+\left(H_{0}-E_{n}^{(0)} \mathbb{1}+\alpha \mathbb{1}\right)^{-1} \phi_{n}\left(\lambda V-\Delta_{n} \mathbb{1}\right)\right]|n\rangle \\
& =\alpha[|c\rangle-M(\alpha) \Delta|n(\alpha)\rangle] .
\end{aligned}
$$

\subsubsection{Finding inverse}

We next multiply the inverse $\left(H_{0}-E_{n}^{(0)} \mathbb{1}+\alpha \mathbb{1}\right)^{-1}$, which is the regularized matrix for the unperturbed eigenvalue equation, to the left on both sides of Eq. (50) to find that

$$
\begin{aligned}
& {\left[\mathbb{1}+\left(H_{0}-E_{n}^{(0)} \mathbb{1}+\alpha \mathbb{1}\right)^{-1} \phi_{n}\left(\lambda V-\Delta_{n} \mathbb{1}\right)\right]|n\rangle} \\
& =\alpha\left(H_{0}-E_{n}^{(0)} \mathbb{1}+\alpha \mathbb{1}\right)^{-1}[|c\rangle-M(\alpha) \Delta|n(\alpha)\rangle] .
\end{aligned}
$$

Now the regularized matrix $\left(H_{0}-E_{n}^{(0)}+\lambda V-\Delta_{n} \mathbb{1}+\alpha \mathbb{1}\right)$ has the inverse and we can solve the regularized eigenvalue equation (44) as

$$
\begin{aligned}
|n\rangle= & {\left[\mathbb{1}+\left(H_{0}-E_{n}^{(0)} \mathbb{1}+\alpha \mathbb{1}\right)^{-1} \phi_{n}\left(\lambda V-\Delta_{n} \mathbb{1}\right)\right]^{-1} } \\
& \times\left[\alpha\left(H_{0}-E_{n}^{(0)} \mathbb{1}+\alpha \mathbb{1}\right)^{-1}\right][|c\rangle-M(\alpha) \Delta|n(\alpha)\rangle] .
\end{aligned}
$$

Because the operator product in front of $[|c\rangle-M(\alpha) \Delta|n(\alpha)\rangle]$ in Eq. (52) is identical to $\alpha M^{-1}(\alpha)$, the second term becomes proportional to $\alpha \Delta|n(\alpha)\rangle$, which vanishes as $\alpha \rightarrow 0$.
At this stage, we can replace $\alpha\left(H_{0}-E_{n}^{(0)} \mathbb{1}+\alpha \mathbb{1}\right)^{-1}|c\rangle$ with $\operatorname{adj}\left(H_{0}-E_{n}^{(0)}\right)|c\rangle$ by rescaling $|c\rangle$ as is done in Eq. (39). Every matrix element of $\operatorname{adj}\left(H_{0}-E_{n}^{(0)}\right)$ is vanishing except for the $n n$ element. In the limit $\alpha \rightarrow 0$, we have

$$
\begin{aligned}
|n\rangle= & {\left[\mathbb{1}+\left(H_{0}-E_{n}^{(0)} \mathbb{1}\right)^{-1} \phi_{n}\left(\lambda V-\Delta_{n} \mathbb{1}\right)\right]^{-1}\left|n^{(0)}\right\rangle\left\langle n^{(0)} \mid c\right\rangle } \\
& \times \prod_{k \neq n}\left(E_{k}^{(0)}-E_{n}^{(0)}\right),
\end{aligned}
$$

where we have used the identity in Eq. (40). We can absorb the factor $\prod_{k \neq n}\left(E_{k}^{(0)}-E_{n}^{(0)}\right)$ into $|c\rangle$ to find that

$|n\rangle=\left[\mathbb{1}-\frac{\phi_{n}}{E_{n}^{(0)}-H_{0}}\left(\lambda V-\Delta_{n} \mathbb{1}\right)\right]^{-1}\left|n^{(0)}\right\rangle\left\langle n^{(0)} \mid c\right\rangle$,

where $\phi_{n}$ is the projection operator defined in Eq. (18). The result is consistent with Eq. (21) if we set $\left\langle n^{(0)} \mid c\right\rangle=1$. In the same manner as we have done with the derivation of Eq. (22) from Eq. (21), we find that

$|n\rangle=\left\{\sum_{k=0}^{\infty}\left[\frac{\phi_{n}}{E_{n}^{(0)}-H_{0}}\left(\lambda V-\Delta_{n} \mathbb{1}\right)\right]^{k}\right\}\left|n^{(0)}\right\rangle\left\langle n^{(0)} \mid c\right\rangle$.

Again, the result is consistent with Eq. (22) if we set $\left\langle n^{(0)} \mid c\right\rangle=1$. From this stage on, we may apply the same procedure to carry out the order-by-order calculation that is given in Sect. 2.4.

\section{Conclusion}

A conventional approach to the time-independent Schrödinger equation with small perturbation is the Rayleigh-Schrödinger perturbation theory which is based on the direct calculation of eigenvalue and wavefunction of a system by explicit series expansions. At lower orders of the series, the formulas are simple, but difficulties arise at higher orders. Another well-known approach is the Brillouin-Wigner perturbation theory, which has a much simpler form at higher orders. However, it has a disadvantage that the wavefunction is expressed in terms of the perturbed energy which must be determined. Except for the two approaches, several methods have been developed so far. We have derived the energy and wavefunction of the perturbed system by making use of a conventional approach for comparison.

We have presented a new formalism to this time-independent Schrödinger equation with small perturbation. This new formalism regularizes the original Schrödinger equation 
with matrix-valued Lagrange undetermined multipliers. The regularization allows one to carry out the inverse transformation that replaces time-consuming procedure of Gaussian elimination $[7,8]$ to obtain the eigenvector. The key mechanism of the new Lagrange-multiplier regularization formalism is to regularize the secular equation that is vanishing in the eigenvalue problem by adding new degrees of freedom to the characteristic matrix. This regularization makes the characteristic matrix invertible and the eigenvector is easily obtained from the inverse of the characteristic matrix, followed by applying the constraint equation. We note that this procedure is similar to adding the gauge-fixing terms to the Lagrange density of gauge fields in gauge-field theories.

We have applied the Lagrange-multiplier regularization formalism to the unperturbed system to demonstrate how it works in solving an eigenproblem. This demonstration will greatly help readers in understanding the mechanism of the Lagrange-multiplier regularization formalism in solving an eigenvalue equation and regularization of the secular determinant with a regularization parameter. With an appropriate normalization factor, we have found that the result is a trivial identity that reproduces the unperturbed eigenket. Another point of this demonstration is that some formulas appearing in the demonstration are actually used in finding the energy and wavefunction of the perturbed system.

Then, we have applied the Lagrange-multiplier regularization formalism to the perturbed system. By regularizing the perturbed Schrödinger equation with matrix-valued Lagrange multipliers and a regularization parameter, we have found the inverse of the corresponding characteristic matrix in the regularized equation. By taking the constraint equation, the eigenvector reduces into the adjugate of the characteristic matrix multiplied by an arbitrary vector. It is remarkable that the regularized characteristic matrix is factorized with a factor of the characteristic matrix in the unperturbed system and it contains all the singular structure of the characteristic matrix in the perturbed system. Finally, we have obtained the energy and wavefunction with all-order corrections in a compact form, which are consistent with those in the conventional approach. This reveals that the method to make use of the Lagrange multipliers does work well in the time-independent perturbation theory and would be very powerful to solve the eigenproblem in physics. In finding the solution, we have never relied on an iterative method. Instead, it is remarkable that we have indeed computed the all-order corrections in a compact form in a straightforward manner.

We have assumed that the Hamiltonian of the unperturbed system is not degenerate. However, the Lagrange-multiplier regularization formalism for perturbation theory that we have developed in this paper can be extended systematically to make it applicable to a degenerate case. One subtle point is that the inverse of the regularized matrix is not expressed in terms of its adjugate matrix. This problem can be resolved by taking into account the diagonal form of the unperturbed Hamiltonian like the proof in appendix A. The degeneracy of eigenstates of the perturbed system are expected to be distinguished, depending on the form of the perturbative potential.

Regularization of a set of indeterminate linear equations by adding new degrees of freedom is a key point of the Lagrangemultiplier regularization formalism, which is powerful and can be applied to a variety of fields in physics and science. The time-independent perturbation theory now is proved to be another concrete example for the applications of the Lagrangemultiplier regularization formalism. Since the Lagrange-multiplier regularization formalism has a more general feature than that in classical mechanics, we believe that the new formalism in this paper will give more insights on not-yet-known applications of Lagrange undetermined multipliers as well as the time-independent perturbation theory in quantum mechanics.

Acknowledgements As members of the Korea Pragmatist Organization for Physics Education $(K P O P \mathscr{E})$, the authors thank the remaining members of $K P O P \mathscr{E}$ for useful discussions. The work of JL and URK is supported in part by Grants funded by the Korea government (MSIT) under Contract No. NRF-2020R1A2C3009918. The work of DWJ and CY is supported in part by Basic Science Research Program through the National Research Foundation of Korea (NRF) funded by the Ministry of Education 2018R1D1A1B07047812 (DWJ) and 2020R1I1A1A01073770 (CY), respectively. The work is also supported in part by the National Research Foundation of Korea (NRF) under the BK21 FOUR program at Korea University, Initiative for science frontiers on upcoming challenges.

\section{Appendix A: Proof of Eq. (41)}

In this appendix, we present a rigorous proof of Eq. (41). The proof of the first identity is given in Ref. [8] and we illustrate the proof of the second identity:

$$
\operatorname{adj}(\mathbb{A})=\operatorname{adj}\left(H_{0}-E_{n}^{(0)} \mathbb{1}\right)=\left|n^{(0)}\right\rangle\left\langle n^{(0)}\right| \prod_{k \neq n}\left(E_{k}^{(0)}-E_{n}^{(0)}\right) .
$$

We define

$\mathbb{A} \equiv H_{0}-E_{n}^{(0)} \mathbb{1}$.

Because $\left|n^{(0)}\right\rangle$ is an eigenket of $H_{0}$ with the eigenvalue $E_{n}^{(0)}$, $H_{0}$ is a diagonal matrix in the basis set $\left\{\left|n^{(0)}\right\rangle \mid n=1,2, \cdots\right\}$. In this basis, the matrix representation for the adjugate matrix $\mathbb{A}=H_{0}-E_{n}^{(0)} \mathbb{1}$ is

$$
\begin{aligned}
\mathbb{A} & =\left(A_{i j}\right)=\left(\left\langle i^{(0)}|\mathbb{A}| j^{(0)}\right\rangle\right) \\
& =\operatorname{diag}\left(E_{1}^{(0)}-E_{n}^{(0)} \cdots E_{n-1}^{(0)}-E_{n}^{(0)} 0 E_{n+1}^{(0)}-E_{n}^{(0)} \cdots\right) .
\end{aligned}
$$


Therefore, the matrix $\mathbb{A}$ can be expressed as

$$
\mathbb{A}=\sum_{k \neq n}\left|k^{(0)}\right\rangle\left\langle k^{(0)}\right|\left(E_{k}^{(0)}-E_{n}^{(0)}\right)
$$

This is a diagonal matrix with the $n n$ element vanishing. Thus the $n$th column and $n$th row are both completely vanishing.

The adjugate of a matrix $\mathbb{A}$ is the transpose of the corresponding cofactor matrix $\mathbb{C}$ :

$\operatorname{adj}(\mathbb{A})=\mathbb{C}^{T}=\left(C_{j i}\right)=\left[(-1)^{i+j} \mathscr{M}_{j i}\right]$.

And the $i j$ element $C_{i j}$ of the cofactor matrix $\mathbb{C}$ is the $i j$ minor $\mathscr{M}_{i j}$ of the matrix A multiplied by $(-1)^{i+j}$. The $i j$ minor $\mathscr{M}_{i j}$ of the matrix $\mathbb{A}$ is the determinant of the submatrix of $\mathbb{A}$ in which the $i$ th row and the $j$ th column are eliminated from $\mathbb{A}$. Except for a single case with $i=j=n, i j$ minor $\mathscr{M}_{i j}$ of the matrix $\mathbb{A}$ is vanishing because there is at least a single null column or a single null row:

$\mathscr{M}_{i j}=0, \quad(i, j) \neq(n, n)$.

The only nonvanishing $i j$ minor is for $i=j=n$ whose value is

$$
\begin{aligned}
\mathscr{M}_{n n}= & \mathscr{D e t}\left[\operatorname { d i a g } \left(E_{1}^{(0)}-E_{n}^{(0)} E_{2}^{(0)}-E_{n}^{(0)} \ldots E_{n-1}^{(0)}\right.\right. \\
& \left.\left.-E_{n}^{(0)} E_{n+1}^{(0)}-E_{n}^{(0)} \ldots\right)\right] \\
= & \prod_{k \neq n}\left(E_{k}^{(0)}-E_{n}^{(0)}\right) .
\end{aligned}
$$

As a result, the cofactor matrix is

$$
\mathbb{C}=\left|n^{(0)}\right\rangle\left\langle n^{(0)}\right| \prod_{k \neq n}\left(E_{k}^{(0)}-E_{n}^{(0)}\right) .
$$

This is a symmetric matrix whose the only nonvanishing element is $C_{n n}$. As a result,
$\operatorname{adj}(\mathbb{A})=\mathbb{C}^{T}=\left|n^{(0)}\right\rangle\left\langle n^{(0)}\right| \prod_{k \neq n}\left(E_{k}^{(0)}-E_{n}^{(0)}\right)$

This completes the proof of Eq. (41).

Author Contributions All authors contributed equally to this work.

Open Access This article is licensed under a Creative Commons Attribution 4.0 International License, which permits use, sharing, adaptation, distribution and reproduction in any medium or format, as long as you give appropriate credit to the original author(s) and the source, provide a link to the Creative Commons licence, and indicate if changes were made. The images or other third party material in this article are included in the article's Creative Commons licence, unless indicated otherwise in a credit line to the material. If material is not included in the article's Creative Commons licence and your intended use is not permitted by statutory regulation or exceeds the permitted use, you will need to obtain permission directly from the copyright holder. To view a copy of this licence, visit http://creativecommons.org/licenses/by/4.0/.

\section{References}

1. D. J. Griffiths, Introduction to Quantum Mechanics, 2nd ed., Pearson Prentice Hall, ISBN-13 : 978-0131118928 (2004)

2. J. J. Sakurai, Modern Quantum Mechanics, Benjamin/Cummings, Inc. (1985)

3. E. Schrödinger, Ann. Physik 80, 437 (1926)

4. W. Silvert, Am. J. Phys. 40, 557 (1972)

5. W.K. Niblack, B.P. Nigam, Am. J. Phys. 38, 101 (1970)

6. L. Lain, A. Torre, Eur. J. Phys. 8, 178 (1987)

7. W. Han, D.-W. Jung, J. Lee, C. Yu, J. Korean Phys. Soc. 78, 1018 (2021)

8. D.-W. Jung, W. Han, U-R. Kim, J. Lee, C. Yu, Finding normal modes of loaded string with lagrange multipliers, J. Korean Phys. Soc. https://doi.org/10.1007/s40042-021-00314-9

9. J.-H. Ee, D.-W. Jung, U.-R. Kim, D. Kim, J. Lee, Eur. J. Phys. 42, $055016(2021)$

Publisher's Note Springer Nature remains neutral with regard to jurisdictional claims in published maps and institutional affiliations. 